\title{
Notch2 inhibits proliferation of chronic myeloid leukemia cells
}

\author{
ZESONG YANG ${ }^{1}$, CHUNXIU YANG $^{2}$, SHUNJUN ZHANG ${ }^{1},{\text { YING } \text { LI }^{3} \text { and JIANBIN CHEN }}^{1}$ \\ ${ }^{1}$ Department of Hematology, The First Affiliated Hospital of Chongqing Medical University, Chongqing; \\ ${ }^{2}$ Department of Hematology, Affiliated Hospital of Zunyi Medical College, Zunyi; ${ }^{3}$ Department of \\ Hematology and Rheumatism, Chongqing Three Gorges Central Hospital, Chongqing, P.R. China
}

Received November 7, 2012; Accepted January 18, 2013

DOI: $10.3892 / 01.2013 .1159$

\begin{abstract}
The Notch signaling pathway has been shown to be involved in the progression of chronic myeloid leukemia (CML). The aim of this study was to investigate the effects of exogenous Notch2 overexpression on cell proliferation and possible mechanisms in the human CML cell line K562. When exogenous intracellular fragment of Notch2 (ICN2) was transfected into K562 cells with Lipofectamine ${ }^{\mathrm{TM}}$ 2000, the expression of Notch 2 mRNA and protein were upregulated. Cell numbers decreased and the proliferation was inhibited significantly after transfection with ICN2. G1 phase cells increased and $\mathrm{S}$ phase cells decreased $48 \mathrm{~h}$ after transfection. Finally, the expression of Numb, Bcl-2, NF- $\kappa \mathrm{B}$ and TGF- $\beta 1$ was detected. It was found that the expression of $N F-\kappa B$ and TGF- $\beta 1$ mRNA was increased, while Bcl-2 was downregulated, with Numb expression unchanged. Our study indicates that the Notch pathway is activated in K562 cells after ICN2 transfection. It inhibited the proliferation of K562 cells, likely by upregulating the expression of $\mathrm{NF}-\kappa \mathrm{B}$ and TGF- $\beta 1$ mRNA and downregulating the expression of $\mathrm{Bcl}-2$.
\end{abstract}

\section{Introduction}

The Notch signaling pathway is a highly conserved evolutionarily signaling pathway which plays an important role in regulating the process of development in species as diverse as Drosophila to humans $(1,2)$. The Notch signaling pathway is composed of a Notch receptor, ligand and CBF1/Su(H)/ Lag-1 family (CSL) DNA binding protein. In mammals, four homolog Notch receptors (Notch 1-4) and five Notch ligands (Jagged 1 and 2, and Delta-like 1, 3 and 4) have been identified. Both Notch receptors and ligands are evolutionarily conserved single-pass transmembrane proteins. After Notch ligands bind to the receptors, the receptor undergoes at least

Correspondence to: Professor Jianbin Chen, Department of Hematology, the First Affiliated Hospital of Chongqing Medical University, Chongqing 400016, P.R. China

E-mail: cqchenjianbin2007@126.com

Key words: chronic myeloid leukemia, Notch signal, gene transfection, cell proliferation two proteolytic cleavage events, releasing the Notch intracellular domain (NICD), which is activated into the cytoplasm, and then translocated to the nucleus, where it binds to the CSL (CBF1 in humans, $\mathrm{RBP}_{\mathrm{J}}$ in mice, Suppressor of Hairless in Drosophila and Lag1 in C.elegans) protein (3). After binding to NICD, CSL turns from a transcription repressor to a transcriptional activator (3). The NICD/CSL complex then recruits the co-activator mastermind-like (MAML) and p300 proteins to form a ternary complex that subsequently activates the transcription of the Notch target genes $(4,5)$. Canonical target genes of Notch include HES1, HES5 and Hey. The activated Notch signaling pathway determines the cell fate, maintains the stem cell state and affects cell proliferation, differentiation, apoptosis, organ formation and morphogenesis (6). In recent years, studies have found that abnormal Notch signaling is involved in tumor formation, hereditary diseases, autoimmune diseases and several other processes (7).

Several studies have demonstrated that Notch signaling is involved in the development of lymphoid leukemias (8), but only a few studies have revealed abnormal Notch expression in myeoloid leukemias. Whether abnormal Notch signaling can result in myelocytic leukemia remains unclear. Certain studies, however, have shown that Notch can affect myelopoiesis, with Notch ligands suppressing the differentiation of progenitor cells to myeloid cells (9). Notch2 overexpression is implicated in the development of chronic B-cell lymphoid leukemias, since B-cells are able to survive significantly longer than normal cells. A study by Ishiko et al found that Notch signaling did not affect the proliferation of the chronic myeloid leukemia cell line K562, but inhibited the development of erythroid/megakaryocytic cells by suppresing GATA-1 activity and inducing apoptosis in cooperation with 12-O-tetradecanoylphorbol-13-acetate (TPA) (10). In contrast, another study demonstrated that activated Notch signaling inhibited the growth of K562 cells, possibly by upregulating expression of $\mathrm{Rb}$ protein (11), although the precise mechanism is not clear.

In this study, K562 cells were used to observe the effects of overexpression of the intracellular domain of Notch2 (ICN2). Overexpression of ICN2 inhibited the proliferation of K562 cells and caused G1 arrest. Notably, the expression level of $\mathrm{NF}-\kappa \mathrm{B}$ and TGF- $\beta 1$ genes were upregulated in K562 cells transfected with ICN2, and Bcl-2 was downregulated, but Numb expression was unchanged. These results may suggest 
that Notch signaling inhibits the proliferation of K562 cells, possibly by regulating gene expression.

\section{Materials and methods}

Cell culture and plasmid transfection. The human CML cell line K562 was propagated in RPMI-1640 medium (Hyclone, Logan, UT, USA) containing 10\% fetal bovine serum (FBS) (Hyclone). The cell lines were grown in a humidified incubator at $37^{\circ} \mathrm{C}$ in the presence of $5 \%$ carbon dioxide. $\mathrm{K} 562$ cells were propagated every two days. One day before transfection, the cell medium was changed to ensure the cells were viable. Log phase growth cells were washed, resuspended in RPMI-1640, plated in 12-well plates at a density of $5 \times 10^{5} / \mathrm{ml}$ and cultured for $1 \mathrm{~h}$. Plasmid pcDNA3.1-ICN2 was transfected into the cells with Lipofectamine ${ }^{\mathrm{TM}} 2000$ according to the manufacturer's instructions (K562-ICN2). Vector pcDNA3.1 was used as control (K562-blank).

Cell proliferation assay. Cells were seeded in 96-well plates at a density of 1,500 cells per well and transfected with ICN. Cell proliferation was assayed after 24 and $48 \mathrm{~h}$ of culture, by incubating in $20 \mu \mathrm{l}$ methylthiazole tetrazolium (MTT) solution. Cells were then incubated at $37^{\circ} \mathrm{C}$ for a further $6 \mathrm{~h}$ when $150 \mu \mathrm{l}$ of dimethyl sulfoxide (DMSO; Sigma, St. Louis, MO, USA) was added to each well and subsequently mixed at room temperature for $10 \mathrm{~min}$. The spectrophotometric absorbance of the supernatant was measured at $550 \mathrm{~nm}$. Each assay was repeated at least three times and data were analyzed with the LSD t-test.

Cell cycle analysis. K562 cells were plated at a density of $\sim 3 \times 10^{5} / 1$ in 12 -well plates and transfected with plasmid for $48 \mathrm{~h}$. Cells were collected, fixed in $1 \%$ methanol-free formaldehyde for $20 \mathrm{~min}$ and subsequently suspended in a $70 \%$ ethanol solution. Cells were then suspended in $1 \mathrm{ml}$ of $0.1 \%$ Triton X-100 solution and incubated in $500 \mu$ propidium iodide solution $(50 \mu \mathrm{g} / \mathrm{ml})$ containing $250 \mu \mathrm{g}$ of DNase-free RNase A. Cells were analyzed using fluorescence-assisted cell sorting (FACS). Each assay was repeated in triplicate and data were analyzed with the LSD-t test.

RNA extraction and semiquantitative reverse transcription $(R T)$-polymerase chain reaction $(P C R)$. Total RNA was extracted from K562 cells with TRIzol reagent (Roche Diagnostics, Mannheim, Germany) according to the manufacturer's instructions. cDNA was synthesized using a kit from Takara with oligo-dT as a primer. PCR was run for 35 cycles with cDNA from $0.1 \mu \mathrm{g}$ of total RNA as a template. The PCR products were resolved on $1.5 \%$ agarose gels and visualized by GoldView staining. The RT-PCR primers used in this study were: human Notch2 (5' sense, TGGTGACCGAGATCCTG AAG; 3' antisense, TTGTTCACAGAGCCTTGTTG); Hes1 (5' sense, TAGCTGATCAGTGGCGTGAC; 3' antisense, ATCATCTGGCCTAGGAGACC); Heyl (5' sense, GAGAGG TCCTCCATTGGAAT; 3' antisense, ATGCACAACAAT GGCAACAG), Numb 5' sense, TACCACGTCCTCACCTG TGG; 3' antisense, TGAAGACTGCAGAACCATTG); Bcl-2 (5' sense, ACCTGACCACTAGCCTCCTG; 3' antisense, GCAGAGCACAGGATTCACAG); TGF- $\beta 1$ (5' sense,
GCCTTGATGGAGAGCTTCAC; 3' antisense, CTTGTG GTGGATGTGGACTG), NF-кB (5' sense, AGTCTGTCC AGGCTCGTCAT; 3' antisense, GGACAGGAAGCTCCT GAATG); and human $\beta$-actin I (5' sense, ACTTGCGCA GAACAAGAGAT; 3' antisense, ACTGCCGCCTTCTCC TTAGA); human $\beta$-actin II (5' sense, GATCTGGCACCA CACCTTCT; 3 ' antisense, AAGGAAGGCTGGAAGAGAGC).

Western blot analysis. To detect the expression of Notch2, whole cell lysates were prepared using cell lysis buffer for western blot analysis and immunoprecipitation [20 $\mathrm{mM}$ Tris ( $\mathrm{pH} 7.5), 150 \mathrm{mM} \mathrm{NaCl}, 1 \%$ Triton $\mathrm{X}-100$, sodium pyrophoshate, $\beta$-glycerophosphate, EDTA, $\mathrm{Na}_{3} \mathrm{VO}_{4}$, leupeptin, $0.1 \mathrm{mM}$ PMSF]. Cell extracts were collected by centrifugation at $13,000 \mathrm{x} \mathrm{g}$ for $8 \mathrm{~min}$ at $4^{\circ} \mathrm{C}$. Protein concentrations of the cell extracts were determined by $\mathrm{BCA}$ protein assay reagents (Beyotime Institute of Biotechnology, Jiangsu, China), according to the manufacturer's instructions. Proteins were resolved by SDS-PAGE on $10 \%$ polyacrylmide gel. The proteins were transferred to a PVDF membrane and detected using immunoblotting. The Notch 2 antibody was incubated with the membrane for $12 \mathrm{~h}$ at $4^{\circ} \mathrm{C}$, and immunoreactive proteins were visualized by incubation with a goat anti-mouse immunoglobulin conjugated to horseradish peroxidase (HRP), and the membrane was developed using Pro-light HRP chemiluminescence reagents from Tiangen Biotech (Beijing) Co., Ltd., Beijing, China. All antibodies were purchased from Santa Cruz Biotechnology Inc. (Santa Cruz, CA, USA).

Statistical analysis. To analyze the data, the mean \pm SD was calculated. One-way ANOVA was used to compare the mean values among multiple groups, and the t-test was applied to compare the mean values between pairs of groups. All data was processed by SSPS 10.0 (SPSS, Inc., Chicago, IL, USA). $\mathrm{P}<0.05$ was considered to indicate a statistically significant result.

\section{Results}

Notch2 gene is overexpressed successfully in K562 cells. To confirm that the plasmid pcDNA3.1-ICN2 was successfully introduced to the K562 cells, RT-PCR and western blot analysis were employed to detect the expression of the gene and protein, respectively. As shown in Fig. 1, the expression of Notch2 mRNA and protein was upregulated significantly, suggesting that ICN2 was successfully introduced into K562-ICN2 cells.

Notch2 signaling is activated in K562 cells. To further assess the activation of the Notch signaling pathway, RT-PCR was used to detect the expression of Notch target gene Hes1, and Heyl of the transfected and control group. As shown in Fig. 2, Hes1 and Hey1 were not expressed in K562-blank cells, but they were expressed strongly in the K562-ICN2 group, indicating that Notch signaling was activated in K562-ICN2 cells.

Overexpression of ICN2 changes the morphology of K562 cells. As observed under an inverted phase contrast microscope (Fig. 3), the plasmalemma of K562-blank cells was 
A

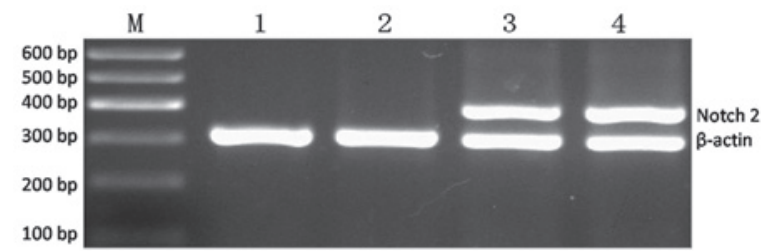

B

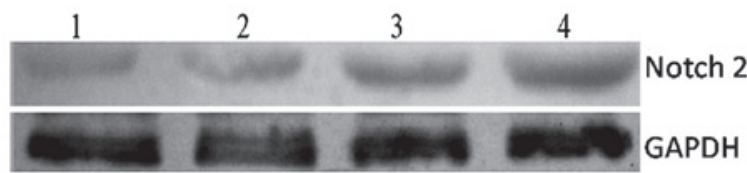

Figure 1. Expression of Notch2 mRNA in K562 cells. M, DNA Marker I; lanes 1 and 2, K562-blank at 24 and 48 h; lanes 3 and 4, K562-ICN2 at 24 and $48 \mathrm{~h}$.

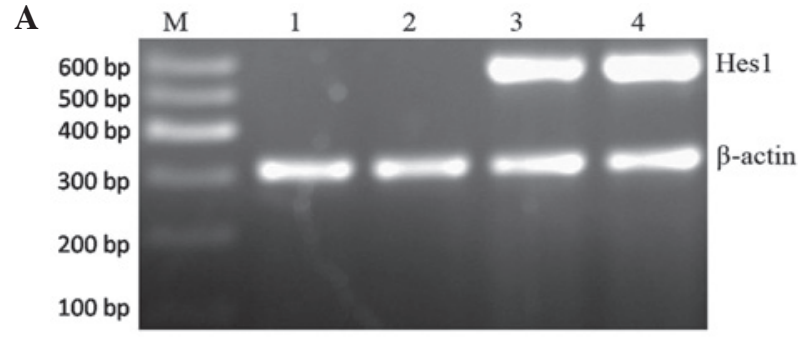

B

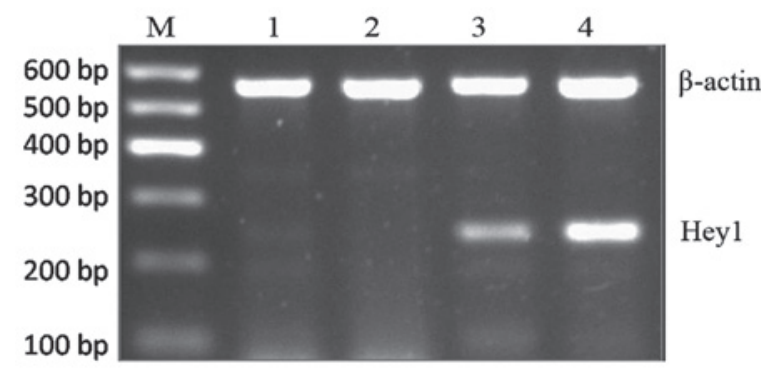

Figure 2. (A) Expression of Hes1 mRNA in K562 cells after transfection with ICN2. M, DNA Marker I; lanes 1 and 2, K562-blank at 24 and $48 \mathrm{~h}$; lanes 3 and 4, K562-ICN2 at 24 and 48 h. (B) Expression of Hey1 mRNA in K562 cells after transfection with ICN2.
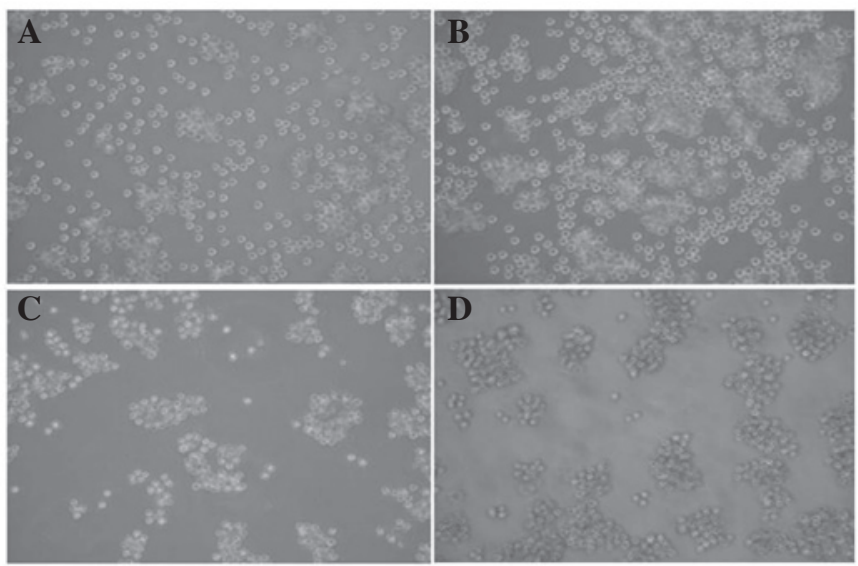

Figure 3. Morphology of K562 cells after transfection with ICN2 under inverted phase contrast microscope (ICC, x400. (A) and (B) K562-blank group at 24 and $48 \mathrm{~h}$; (C) and (D) K562-ICN2 group at 24 and $48 \mathrm{~h}$.
日 K562-blank m K562-ICN2

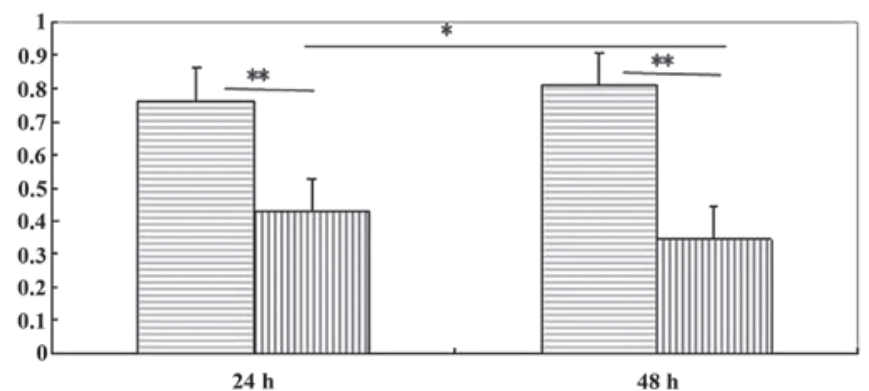

Figure 4. Cell proliferation of K562 cells after transfection with ICN2. ${ }^{*} \mathrm{P}<0.05,{ }^{, * *} \mathrm{P}<0.01$.
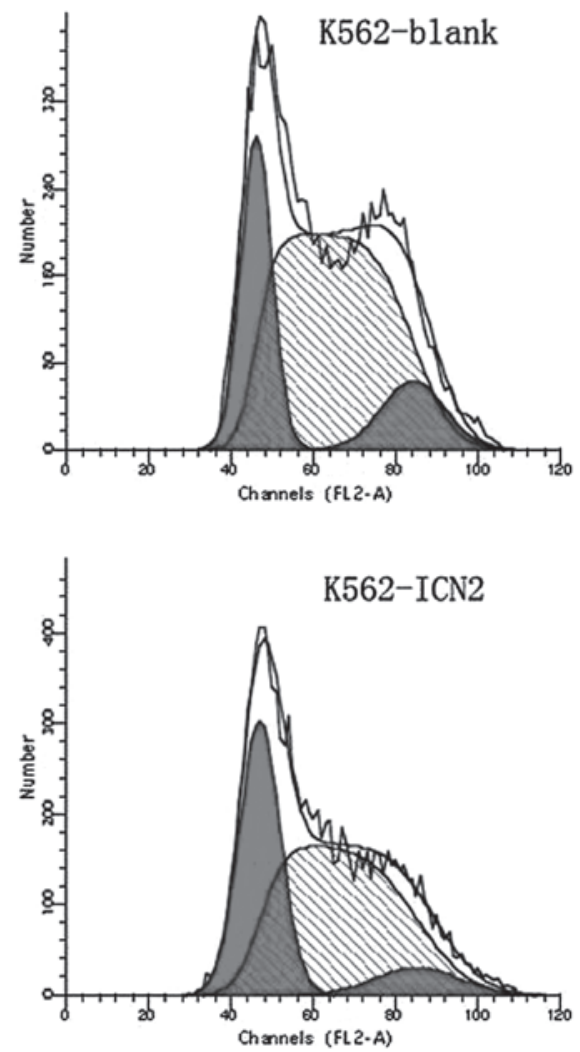

Figure 5. Cell cycle results of K562 cells.

transparent. The longer the culture time, the larger the cell number. In contrast, the K562-ICN2 cells were aggregated when cultured for $24 \mathrm{~h}$ with small cells with a cloudy plasmalemma. The longer the culture time the smaller the cell diameter and volume.

Activation of Notch by transfection with ICN2 inhibits the proliferation of $K 562$ cells. To determine whether Notch signaling affects the proliferation of K562 cells, the growth of K562-ICN2 cells was compared with the K562-blank cells using the MTT assay. As shown in Fig. 4, the growth of K562-ICN2 cells was considerably slower than that of the K562-blank cells $(\mathrm{P}<0.01)$. These data suggest that overexpression of the constitutively active Notch signaling could inhibit the proliferation of the human CML cell line K562 in vitro. 
Table I. Cell cycle change of K562 cells after transfection with ICN2 for $48 \mathrm{~h}$ (mean \pm SD, \%).

\begin{tabular}{llll}
\hline Group & G1 phase & S phase & G2+M phase \\
\hline K562-blank & $21.52 \pm 2.51$ & $63.37 \pm 3.44$ & $10.82 \pm 0.96$ \\
K562-ICN2 & $39.17 \pm 1.50^{\text {a }}$ & $32.94 \pm 3.75^{\text {a }}$ & $13.41 \pm 1.97$ \\
\hline
\end{tabular}

${ }^{\mathrm{a}} \mathrm{P}<0.01$; compared with the control group.

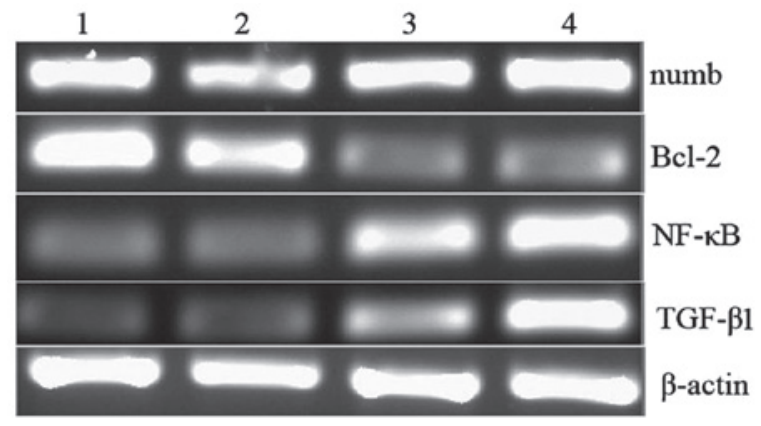

Figure 6. Expression of related genes in K562 cells after transfection with ICN2. Lanes 1 and 2, K562-blank at 24 and 48 h; lanes 3 and 4, K562-ICN2 at 24 and $48 \mathrm{~h}$.

Notch activation rearranges the distribution of the cell cycle of $K 562$ cells. The effect of transfection with ICN2 for $48 \mathrm{~h}$ on the course of the cell cycle using flow cytometry was investigated. As shown in Fig. 5 and Table I, K562 cells showed a G1 arrest and there were fewer $\mathrm{S}$ phase cells.

Activation of Notch signaling pathway regulates the expression of certain genes in $K 562$ cells. The molecular mechanism underlying the growth-regulatory effect of Notch signaling on K562 was investigated. The expression of the molecules related with cell proliferation in K562 cells was measured. RT-PCR analysis showed that the expression of NF- $\kappa \mathrm{B}$ and TGF- $\beta 1$ was upregulated, and that the expression of $\mathrm{Bcl}-2$ was downregulated. The expression of Numb, however, was unchanged.

\section{Discussion}

Different Notch receptors have varying functions in the development of tumors, depending on the cell context. Notch1 has been shown to inhibit the cell growth of hepatic carcinoma, small cell lung cancer and prostate cancer in a previous study (7). When all four Notch receptors are activated they inhibit the growth of acute B-cell lymphoid leukemia and induce apoptosis (12). In contrast, one study has proven that overexpression of Notch 3 promots the growth of human lung cancer cells in vitro and inhibits the differentiation of lung cancer cells in transgenic mice. Notch3 is expressed at a high level in human pancreatic cancer cells and acute T-cell lymphoid leukemia cells. The mechanism of the Notch receptor function as an oncogene or tumor suppressor gene remains to be elucidated.
An important member of the Notch signaling family is the Notch2 receptor, which plays a key role in regulating developmental processes in the embryo (13). A study has shown that Notch2 was upregulated in non-small cell lung carcinoma (NSCLC) and promoted the proliferation of NSCLC cells (14). Expression of Notch2 is necessary for marginal zone B-cell development and is related to CD23 overexpression in chronic B-cell lymphoid leukemia (15). Overexpression of ICN2 can induce T-cell lymphoid leukemia and cells are more easily prone to becoming CD8 cells when expression of Notch2 is inhibited. Conversely, Notch2 is a new tumor suppressor; it inhibits tumor progress in human breast cancer and small cell lung cancer (16). In this study, activation of the Notch2 signal pathway significantly inhibited the proliferation of K562 cells and changed the cell morphology. These results suggest that Notch2 gene is a potential tumor suppressor in chronic myeloid leukemia.

Tumor cells cleave fast and have a high proliferation ability. G1 phase is a DNA presynthetic phase of cells which determines the cell cycle. A recent study found the p53 gene product is an important factor in the regulation of the cell cycle and apoptosis, and p53 accumulated quickly when DNA was damaged by extrinsic factors and G1 arrest occurred (17). Notch 2 inhibited the proliferation of K562 cells and may simulate the function of p53 to induce G1 arrest.

The crosstalk between Notch and other signaling pathways is complicated, and the physiological correlation remains unclear. Numb was considered to be a security device of WNT-Notch signal transduction pathway. The silence, loss of function or mutation of this gene can induce tumorigenesis (18). A study has found that there is crosstalk between Notch and NF- $\kappa \mathrm{B}$ when their roles in normal development and cancer formation are considered (19). Transfected Notch1-ICD in the mouse T-ALL cell line stimulates NF- $\kappa$ B expression through a canonical pathway. A study on a human cervical cancer cell line found that Notch1 activates NF- $\kappa \mathrm{B}$ by interacting with the I $\kappa \mathrm{B}$ kinase (IKK) signal. This occurred in both the cytoplasm and the nucleus (20). The Bcl-2 family of proteins determined the mitochondrial events in cell death and mediated the apoptosis induced by a numner of stimulants (21). Bcl-2 is expressed at a high concentration in many tumor cells and is involved in drug resistance. TGF- $\beta 1$ is a pleiotropic anti-inflammatory factor, which regulates T-cell differentiation. When $\gamma$-secretase inhibitor (GSI) inhibits Notch signaling, TGF- $\beta 1$ induces Foxp 3 expression and naive T-cell proliferation is blocked (22). TGF- $\beta 1$ inhibits the growth of normal cervical cells causing G1 arrest and apoptosis (23). 
In this study, it was identified that overexpression of ICN2 upregulated the expression of NF- $\kappa \mathrm{B}$ and TGF- $\beta 1$, and downregulated the expression of $\mathrm{Bcl}-2$, while the expression of Numb was unaffected. These results suggest that enhanced expression of Notch2 inhibits cell proliferation, suggesting a pathway which may affect the cell cycle distribution by upregulating the NF- $\kappa \mathrm{B}$ and TGF- $\beta 1$ genes and downregulating $\mathrm{Bcl}-2$. Further research is required to confirm our findings and to elucidate the mechanism of Notch in chronic myeloid leukemia.

\section{References}

1. Artavanis-Tsakonas S, Rand MD and Lake RJ: Notch signaling cell fate control and signal integration in development. Science 284: 770-776, 1999.

2. Greenwald I: LIN-12/Notch signaling: lessons from worms and flies. Genes Dev 12: 1751-1762, 1998.

3. Mumm JS and Kopan R: Notch signaling: from the outside in. Dev Biol 228: 151-165, 2000.

4. Jarriault S, Le Bail O, Hirsinger E, et al: Delta-1 activation of Notch-1 signaling results in HES-1 transactivation. Mol Cell Biol 18: 7423-7431, 1998.

5. Kuroda K, Tani S, Tamura K, et al: Delta-induced Notch signaling mediated by RBP-J inhibits MyoD expression and myogenesis. J Biol Chem 274: 7238-7244, 1999.

6. Ohishi K, Katayama N, Shiku H, et al: Notch signaling in hematopoiesis. Cell Dev Biol 4: 143-150, 2003.

7. Leong KG and Karsan A: Recent insights into the role of Notch signaling in tumorigenesis. Blood 107: 2223-2233, 2006.

8. Pancewicz J, Taylor JM, Datta A, et al: Notch signaling contributes to proliferation and tumor formation of human T-cell leukemia virus type 1-associated adult T- cell leukemia. Proc Natl Acad Sci USA 107: 16619-16624, 2010.

9. de Pooter RF, Schmitt TM, de la Pompa JL, et al: Notch signaling requires GATA-2 to inhibit myelopoiesis from embryonic stem cells and primary hemopoietic progenitors. J Immunol 176 5267-5275, 2006.

10. Ishiko E, Matsumura I, Ezoe S, et al: Notch signals inhibit the development of erythroid/megakaryocytic cells by suppressing GATA-1 activity through the induction of HES1. J Biol Chem 280: 4929-4939, 2005.
11. Yin DD, Fan FY, Hu XB, et al: Notch signaling inhibits the growth of the human chronic myeloid leukemia cell line K562. Leuk Res 33: 109-114, 2009.

12. Zweidler-McKay PA, He Y, Xu L, et al: Notch signaling is a potent inducer of growth arrest and apoptosis in a wide range of B-cell malignancies. Blood 106: 3898-3906, 2005.

13. Chen W, Jiang H, Wang M, et al: Effects of chlorpyrifos exposure on kidney Notch2-Jagged1 pathway of early prenatal embryo. Birth Defects Res B Dev Reprod Toxicol 92: 97-101, 2011.

14. Bastide K, Uqolin N, Levalois C, et al: Are adenosquamous lung carcinomas a simple mix of adenocarcinomas and squamous cell carcinomas, or more complex at the molecular level? Lung Cancer 68: 1-9, 2010.

15. Gibb DR, EI Shikh M, Kang DJ, et al: ADAM10 is essential for Notch2-dependent marginal zone B cell development and CD23 cleavage in vivo. J Exp Med 207: 623-635, 2010.

16. Mazur PK, Grüner BM, Nakhai H, et al: Identification of epidermal Pdx1 expression discloses different roles of Notch1 and Notch2 in murine Kras(G12D)-indenced skin carcinogenesis in vivo. PloS one 5: e13578, 2010

17. Yang PM, Huang WC, Lin YC, et al: Loss of IKKbeta activity increases p53 stability and p21 expression leading to cell cycle arrest and apoptosis. J Cell Mol Med 14: 687-698, 2010.

18. Cheng X, Huber TL, Chen VC, et al: Numb mediates the interaction between Wnt and Notch to modulate primitive erythropoietic specification from the hemangioblast. Development 135: 3447-3458, 2008.

19. Wang Z, Banerjee S, Ahmad A, et al: Activated K-ras and INK4a/Arf deficiency cooperate during the development of pancreatic cancer by activation of Notch and NF- $\kappa B$ signaling pathways. PloS One 6: e20537, 2011.

20. Song LL, Peng Y, Yun J, et al: Notch-1 associates with IKKalpha and regulates IKK activity in cervical cancer cells. Oncogene 27: 5833-5844, 2008.

21. Frenzel A, Grespi F, Chmelewskij W, et al: $\mathrm{Bcl} 2$ family proteins in carcinogenesis and the treatment of cancer. Apoptosis 14: 584-596, 2009.

22. Morita Y, Ismail DM, Elkon KB, et al: Dichotomous response to transforming growth factor $\beta$ after $\mathrm{T}$ cell receptor activation by naive CD4+ T cells from DBA/1 mice: enhanced retinoic acid receptor-related orphan nuclear receptor $\gamma$ t expression yet reduced FoxP3 expression. Arthritis Rheum 63: 118-126, 2011.

23. Rorke EA, Zhang D, Choo CK, et al: TGF-beta-mediated cell cycle arrest of HPV16-immortalized human ectocervical cells correlates with decreased E6/E7 mRNA and increased p53 and p21(WAF-1) expression. Exp Cell Res 259: 149-157, 2000. 